\title{
Quantification of Particle Size and Concentration Using In-line Techniques and
}

\section{Multivariate Analysis}

\author{
Carla Ferreira $^{1,2^{*}}$, Javier Cardona ${ }^{2,3}$, Okpeafoh Agimelen ${ }^{1,2}$, Christos Tachtatzis ${ }^{3}$, Ivan \\ Andonovic $^{3}$, Jan Sefcik ${ }^{1,2}$ and Yi-Chieh Chen ${ }^{2, *}$ \\ ${ }^{1}$ EPSRC Future Manufacturing Research Hub for Continuous Manufacturing and Advanced \\ Crystallisation (CMAC), University of Strathclyde, Glasgow, United Kingdom \\ ${ }^{2}$ Department of Chemical and Process Engineering, University of Strathclyde, Glasgow, United \\ Kingdom \\ ${ }^{3}$ Department of Electronic and Electrical Engineering, University of Strathclyde, Glasgow, United \\ Kingdom \\ e-mail: yichieh.chen@strath.ac.uk \\ Tel: +44 1415745304
}

\begin{abstract}
We study means of extracting quantitative information about particle attributes using state-ofart in-line and off-line particle measurements and analysis techniques. The approach comprises a combination of image analysis, laser diffraction, inversion of chord length distribution, and multivariate analysis. Polystyrene particle suspensions are used as the model system to provide a wide range of particle loadings (up to $10 \mathrm{wt} \%$ ), sizes $(<90$ to $800 \mu \mathrm{m})$ and shapes. We identify key challenges and limitation of the in-line imaging and chord length measurements; particularly, an upper limit of particle number density of $10,000 \mathrm{~g}^{-1}$ is observed, as well as the impact of internal reflections from large and transparent particles. The latter phenomena deteriorate the accuracy of the chord length distribution and the subsequent particle size estimation using inversion algorithms. The study demonstrates the use of multivariate analysis
\end{abstract}


for quantifying particle size and concentration, which yields relative errors of 6 and $11 \%$, respectively.

Keywords: particle size distribution; solid concentration; chord length distribution; in-line quantitative particle analysis; partial least square regression analysis;

\section{Introduction}

The ability to monitor key quality attributes in pharmaceutical, chemical and biological processes in real-time has been the key motivation that drives the development of Process Analytical Technologies (PAT). In-line analysis methods offer fast, non-destructive and nondisruptive measurements, presenting an attractive alternative to off-line options. For example, particle size and shape are critical physical attributes in the pharmaceutical industry and have a direct impact on downstream process efficiency, $[1,2]$ and product quality.[3, 4] The key benefit of obtaining real-time information from in-line particle analysers is to enable real-time feedback/feedforward process control, a major contribution to optimal process operation, process/product consistency and critical to the migration from batch to continuous manufacture. In-line analysers are typically configured with reduced measurement flexibility and tighter control of the measurement parameters. For complex scenarios where particulate substances evolve significantly with the process and/or vary with process conditions, the selection of a suitable in-line particle analyser for the entire process is not straightforward. To date, only a few techniques are capable of in situ/in-line analysis for real-time process control and optimisation, and little has been reported on the comparison of the performance and reliability of different particle analysis methods.[5-7] Most studies using in-line particle analysers only utilise the measurement as a quality indicator. Spectroscopic-based techniques require indirect inference of crucial properties of particles from the light scattering effect on spectra.[8-10] The 
capability of in-line measurement techniques to quantify key particle attributes has not been subject to extensive studies; state-of-the-art techniques typically involve the analysis/inversion of the measurement using physical-based models, which are time-consuming and often do not account for interferences that occur in practice. The ability for in-line particle analysers to provide reliable and real-time information about the process is highly desirable to meet the growing trend in adopting PAT in manufacturing. Achieving this aim requires the fundamental understanding of different analysers and a reliable approach to deriving the information from the captured measurements.

\subsection{Off-line particle analysis - sieving, laser diffraction and microscopy}

Traditionally, particle size measurements have been conducted using off-line techniques such as sieving, laser diffraction (LD) and microscopy. Sieving is capable of separating particles in a broad size range and is easy to implement; the diameter of the particle is defined as the smallest lateral dimension of the particle which passes through a given sieve size. Sieving is most accurate and effective for spherical or near-spherical particles; particles of non-spherical shape often lead to anomalous results.

Laser diffraction (LD) is a widely accepted standard method in many industries for the measurement of a wide range of particle types and sizes, typically between 0.01-3000 $\mu \mathrm{m}$, and usually applies to particles above $1 \mu \mathrm{m}$.[11] LD measures the scattering pattern from dispersed individual particles illuminated by a laser beam.[5] The analysis of the scattering pattern assumes that all particles are spherical and typically requires diluting samples, a process that can alter the characteristics of the particles and is difficult to apply in-line for most applications. Microscopy image analysis allows the assessment of two-dimensional particle size and shape information; in the case of non-spherical particles, the preferential dimensions of particles are the two longer axes. Therefore, analysis of 3D size and shape population of a non-spherical particle is skewed and often used along with non-visual techniques to mitigate such bias.[12] 
Although the interpretation of the results from the off-line analysis is usually more straightforward, errors introduced during sampling, sample preparation or transport can affect the representativeness of the results. Furthermore, the consequences of delayed detection can be costly, and can even lead to the discard of the product.

\subsection{In-line particle analysis - imaging and chord length measurement}

The most common techniques for in-line particle measurement include particle Chord Length (CL) measurement, microscopic imaging, and spectroscopy. Chord lengths (CLs) of particles, defined as the distance between any two edges of a particle's 2-D projection, have been utilised to infer particle size.[13-15] CL measurements acquire the duration of the signals reflected by particles passing in front of a laser illumination beam. The statistical representation of the multiple CLs detected is presented in the form of chord length distribution (CLD). Several methods have been developed to extract particle size information from a CLD; however, the ill-posed nature of the inversion presents a significant challenge to yielding a reliable solution.[16-19] Within the accuracy of experimental data, the same CLD can potentially correspond to multiple combinations of the population distribution of particle size and shape.

Similar to the off-line approach, in-line imaging method returns a 2-D projection of particles. Many optical imaging systems have been developed, including Photometric Stereo Imaging,[20] Eyecon technology,[21] stereoscopic imaging[22] and Particle Vision Microscope (PVM).[23] The in-line approach captures the projection of free-flowing particles in the optical plane of the instrument. However, the accuracy of the particle analysis is a function of the instrument's optical volume, which depends on its optical setting. For example, images of partially in-focus particles may be mistakenly treated as smaller particles by the analysis algorithm, compromising the accuracy of the interpretation.[24]

Although in-line particle size analysers are not designed for assessing particle concentration, the count of particles in images or CL is related to the number of particles in a system and can 
act as an indicator. Furthermore, the ill-posed issue for inverting particle size information from CLDs constrains the CL measurement for qualitative assessment of particle attributes. Understanding how to best utilise the information captured by a PAT tool is important in maximising the knowledge on crucial attributes in processes, as well as to develop a robust inline analysis strategy.

Multivariate regression analysis (MVA) has been applied to CLDs to model, for example, the relationship of particle size during granulation with the process parameters used to establish the granulation endpoint and to determine cake resistance.[25-27] However, no methods have been reported to obtain particle size information directly.

Here, means of extracting quantitative information on particle size and concentration from commercial in-line and off-line particle measurement techniques are presented. Suspensions of polystyrene particles of different sizes and shapes at different solid loading are used as a model system. In the case of CL measurements, partial least square regression (PLSR) analysis is applied to overcome the limitations of CLD inversion approaches, providing a robust means of quantifying particle size and concentration.

\section{Material and methods}

\subsection{Materials and experimental setup}

Polystyrene (PS) particles were used as the model system due to the broad range of particle size and shape that can be synthesized using suspension polymerisation reactions. [28] Particles were sieved into six size groups from $<90 \mu \mathrm{m}$ to $800 \mu \mathrm{m}$ with shapes varying gradually from ellipsoids to spheres. Particles in each group were suspended in deionized (DI) water at five solid loadings: $1.0,2.5,5.0,7.5$ and $10 \mathrm{wt} \%$, yielding a total of 30 samples of different size and concentration. Table 1 summarises the size range of PS particles and their respective nomenclature used in the study. 
For in-line measurements, polystyrene suspensions were placed in a $250 \mathrm{ml}$ glass jacketed tank reactor (Radleys, UK), as shown in SFigure 1 in the Electronic Supplementary Information (ESI). The reactor was fitted with a PTFE turbine stirrer of $40 \mathrm{~mm} ø, 315 \mathrm{~mm}$ (long ø shaft) and operated clockwise at $500 \mathrm{rpm}$. In-line imaging and chord length measurement probes were positioned at an inclination of $30^{\circ}$. Stirring speed, probe direction and position were optimised to mitigate interference between optical measurements.

\subsection{In-line and off-line measurements}

In-line imaging. Images of the particles in suspension were captured using a PVM V819 probe (Mettler Toledo, UK). Greyscale images were acquired from a field of view of $1075 \mu \mathrm{m} \times 825$ $\mu \mathrm{m}$. Each image frame consists of $1360 \times 1024$ pixels with a pixel size of $0.8 \mu \mathrm{m}$. Diffuse transmission images were obtained using a Teflon reflector cap attached to the probe.

The processing of the particle images follows the procedure described by Cardona et al.[24] The image analysis algorithm only accepts particles in focus and larger than $24 \mu \mathrm{m}$, and the results are expressed in the form of Equivalent Circle Diameter (ECD).[29] 10,000 frames of images for each sample are analysed; at least 500 in-focus particles are required to form a valid PSD. In this study, the results are expressed in number- and volume-weighted PSDs. The algorithm also provides aspect ratio distributions, as described in Ref. 24.

In-line chord length (CL) measurement. CL measurements were conducted using an FBRM G400 system (Mettler- Toledo, UK) which expresses the CLD as a distribution containing 100 geometrically-spaced bins. Considering the broad range of particle sizes analysed and the requirement for quantitative comparison with other particle measurement techniques, CLDs acquired using the macro mode were chosen for further analysis, including unweighted and square-weighted CLDs. The former was further inverted into a volume-weighted PSD using the algorithm developed by Agimelen et al., which utilizes the Fredholm integral equation (first 
kind).[16, 30-32] The algorithm assumes the same aspect ratio for all particles, and the size is expressed as ECD.

Off-line imaging. The static microscope-imaging analysis was performed using Morphologi G3 (Malvern Instruments Limited, UK) to measure particles size and shape. PS particles were dispersed using an integrated dry powder disperser, and images of individual particles were captured using a high-resolution digital camera. The setup typically measures particles of size between $0.5 \mu \mathrm{m}$ to $1000 \mu \mathrm{m}$.

The system utilises advanced graphing and data classification software to analyse at least 500 particles per PS group and obtain representative particle distribution statistics. Anomalous objects such as fibres, aggregated particles and fines, were discarded using the visualisation tool provided. The results of the analysis are displayed as Equivalent Circle Diameter (ECD) and aspect ratio distributions. The aspect ratio is defined as the ratio of the shortest to the longest axis of a particle. It can vary from nearly zero for very elongated and thin particles to one for spherical particles.

Off-line laser diffraction (LD). The LD measurements were conducted using a Mastersizer 3000 (Malvern Instruments Limited, UK). PS particles were dispersed in DI water using a Hydro MV cell (Malvern Instruments Limited, UK) by adding particles to the cell to reach a laser obscuration of $15 \%$. Five measurements were taken for each sample. LD measurements are expressed as the volume-weighted distribution of Equivalent Sphere Diameter (ESD).

\subsection{Data representation and multivariate regression analysis}

Particle size distribution (PSD) representation. Due to the nature of the measurement principles, each particle size instrument has a preferred mode of expressing PSD, and their direct comparison is not straightforward. Table 2 summarises the different size ranges and the number of bins for each technique; ESD and ECD are only comparable for spherical particles. 
Depending on the measurement principle and the shape of non-spherical particles, ECD and ESD exhibit different discrepancies.

Furthermore, since each technique uses different size range, the number of bins and width of the bin to express the PSD, all PSDs were converted into probability density functions (PDF) to allow a grid-independent comparison for all measurements. The PDF is the probability of a random variable falling within a particular range of values which can be calculated from the area under the PDF curve.[33]

Partial least square regression (PLSR) analysis. PLSR analysis was applied to the unweighted and square-weighted CLDs to extract quantitative information on particle size and concentration. The analysis uses the algorithms reported by Chen et al.[34] The median particle size, D50, from LD and concentration from gravimetric measurements were used as the reference values for the model. PLSR model performance was tested using leave-one-out crossvalidation, and the merit of the models was obtained based on the number of latent variables (LVs), the root-mean-square error of cross-validation (RMSECV), RMSECV curves and the correlation coefficient $\mathrm{R}^{2}$. The loading curves and scores of the latent variables used in the models were also considered, as well as the randomness of the residual plot. Two approaches, $\log$ transformation and Standard Normal Variate (SNV) pre-processing,[35] were used to preprocess the CLDs into a comparable scale prior to establishing the model. All data, with or without pre-processing, was mean centred prior to model formation.

\section{Results and Discussion}

To determine the performance of different methods at extracting quantitative particle information, the effect of particle size and concentration on PSDs are analysed. The causes of the discrepancies between measurement techniques are discussed, along with their main 
strengths and limitations. The goal is to examine the effectiveness of extracting particle size and concentration from the measured signal. In the case of CLD measurements, PLSR models are established to improve the estimation of particle size and concentration.

\subsection{Effect of particle size in off-line \& in-line PSD}

For consistency, the comparison is conducted using volume-weighted PSDs for all techniques considered. The comparison of grid-independent PDFs from in-line and off-line imaging, LD and CL techniques is shown in Figure 1; for the in-line measurement techniques, the PDFs are estimated using measurements on $10 \mathrm{wt} \%$ particle suspensions to ensure sufficient sampling of particles. All PDFs are broader than the range defined by sieving due to the variety of particle shapes in the sample. Figure 2(a)-(f) present typical images captured using the in-line imaging technique, clearly indicating the presence of a mix of ellipsoidal and spherical particles for the majority of the particle size groups. As non-spherical particles like ellipsoids can pass a sieve mesh of an aperture smaller than the length of its long axis, the sieve diameter does no longer correspond to the ESD. In this case, using sieving to determine particle size range can lead to an underestimation bias of particle size.[36-38]

Different anomalies between techniques can be observed for each particle size group. For example, in-line and off-line imaging techniques do not result in similar PDFs, particularly in Figures 1(a), (b) and (e). The images are further analysed to determine the cause of the anomalies. The cumulative distribution of aspect ratios for both in-line and off-line imaging methods is shown in Figure 2(g). Both cases indicate a decrease of ellipsoidal particles in the sample for groups of large particle size, as evidenced by the in-line images in Figure 2(a)-(f). In general, a larger proportion of elongated objects is observed through off-line measurement. How accurately the axes of non-spherical particles are described depends on the imaging technique. For example, the shortest axis of platelet-like particles is often under-represented in off-line image analysis due to the two longest axes being preferred for static particles on 
microscope slides. On the other hand, in-line imaging techniques are known to be more susceptible to the presence of out-of-focus particles. The in-line method is likely to capture images of partially focused particles at different orientations; constructing the true shape of the non-spherical particles using in-line imaging is thus challenging.[24] Instead, the analysis could interpret out-of-focus particles as images of smaller particles, causing a shift of the PDFs towards smaller sizes with respect to off-line imaging observed in Figure 1(a) and (b).

The effect of partially focused and randomly oriented particles in in-line image analysis is also evident in Figure 2(g). Observing the longest dimensions of the particle in the in-line analysis is unlikely since moving particles orient randomly with respect to the field of view of the camera, leading to over-estimation of the aspect ratio, as observed in Figure 2(g). In this circumstance, only a fraction of the ellipsoidal particles happens to orient at a position that projects their longest axis in the $2 \mathrm{D}$ image. The rest of the ellipsoidal particles orient at an angle to the image plane, subsequently, leading to particle images with a shorter long axis. Unlike the off-line method, where particles fall into two distinctive aspect ratio groups, $\sim 0.2-0.5$ (ellipsoids) and 1 (spheres), the cumulative distribution of aspect ratio increases gradually for in-line image analysis with a smaller particle percentage for the smallest aspect ratio, as shown in Figure 2(g) for the samples containing ellipsoidal particles (PS0-90 to PS250-300). The findings in Figure 2 suggest that off-line image analysis is more sensitive in resolving the particle population of different shapes. The PDF peaks for the off-line method in Figure 1(b) indicate the contribution of the spherical and ellipsoidal particles to the ECD. In contrast, the in-line method results in broadening of the PDF and the appearance of a shoulder. Better agreement in PSD between the in-line and off-line imaging techniques is observed from samples that mostly consist of spherical/nearly spherical particles (see Figures 1(c)-(e)).

The under-estimation of the population size in Figure 1(e) by the in-line method is related to particles' size reaching the dimensions of the image frame; the image analysis algorithm 
discards particles that cross the image frame to avoid biasing results. Larger particles, which are more likely to fall on the image frame, will, therefore, be under-represented in the PDF. In fact, for the largest particles, i.e. PS630-800, it was not possible to capture enough particles to provide a representative population from the 10,000 frames acquired.

All PDFs from the LD method exhibit a broad and smooth mono-modal ESD distribution, despite the presence of ellipsoids identified by imaging techniques. In general, the PDFs extracted from LD tend to shift towards smaller ESD when compared with these techniques owing to the method of inversion of the diffraction pattern averaged across different particles. In the case of ellipsoidal particles, the diffraction pattern of the short axis of the particles is more likely to be measured, which subsequently contributes to the skewed PDF curves towards smaller sizes.[39] As the population of spherical particles increases, the consistency between the imaging and the LD method increases.

Many challenges are reported for estimating reliable PSDs from CLDs; the results in Figure 1 demonstrate some of these limitations. For instance, at high solid loading, the method may collect CLs from overlapped particles instead of individual particles, as suggested by the overcrowded particle images in Figure 1(a). The over-lapped particle group results in unresolvable longer CLs, which subsequently translates in larger particle sizes. Increasing particle size at a fixed solid loading reduces the particle number density, improving the resolution of CL measurement, as observed in Figures 1(b) and (c). Another limitation of CL measurements is the interference of internal reflection from the transparent polystyrene beads. The inverted PSDs in Figure 1(d) and (e) exhibit a peak at around $150 \mu \mathrm{m}$, related to the internal reflection of the incident laser by the particle, as also observed by the in-line imaging probe for a particle illuminated directly (in SFigure 2 in ESI). The CL measurement suffers from specular reflection and additional internal scattering which splits the length of the chords.[40-43] As inline imaging indicates that particles in all size groups are transparent, such interference is 
expected to affect all CLDs and their corresponding inverted PSDs and results in the persistent peak of smaller size. Increasing particle size leads to the increased separation of the split chords, and eventually becomes well-resolved in the inverted PSD as demonstrated in Figure 1(e). For PS630-800 (Figure 1(f)), the measurement is severely compromised by chord splitting, which affects most of the particles since no contribution is observed at the expected particle size.

The comparison in Figures 1 and 2 highlights the advantages and shortcomings of different particle sizing techniques. The presence of non-spherical particles poses a significant challenge to the off-line LD and in-line - both imaging and chord length - measurements; transparent particles further deteriorate the performance of chord length analyses. Results also highlight the challenges in analysing samples with high particle density, discussed in detail in the next section.

\subsection{Effect of solid loading on in-line measurements}

The effect of solid loading is investigated using only in-line methods as the off-line methods are not suitable to quantify particle population in suspensions due to sample preparation requirements. Although PDFs cannot be utilised to quantify particle content as PDFs are normalised, an increase of solid loading can manifest as an increase in the number of particles imaged or chord lengths captured per unit time. This offers an opportunity to utilise CL and imaging techniques to determine the solid content of a suspension.

Figure 3 uses the results for PS125-180 to demonstrate typical changes in PSDs from the imaging method (in (a) and (b)) and in CLDs (in (c) and (d)). Similar to those observed in Figure 1(b), the volume-weighted PSDs in Figure 3(b) exhibit a main and a shoulder peak corresponding to the spherical and ellipsoidal particles in the sample, respectively. Instead of monotonically increasing with increasing solid loading, the particle counts in the PSDs show 
an initial increase, followed by a decrease at higher solid loadings. Analysis on other particle size groups shows similar responses; the particle count only follows the increase of solid loading at low concentrations (SFigure 3 in ESI). On the other hand, a gradual increase in counts is clearly observed in all CLs for both unweighted and square-weighted CLDs of PS125180, as shown in Figure 3(c) and (d). In addition, in both cases, CLDs exhibit a shift of the distribution towards smaller CLs, merging of the shoulder (at around $70 \mu \mathrm{m}$ ) with the main peak. In the case of unweighted CLDs, a similar response is consistently observed for other particle size groups (SFigure 4).

As shown in Figure 2(a)-(f), the crowded particle population at high solid content can form overlapped particle images. It suggests that, in the CL measurement, the CL from a particle could become inseparable from the CLs corresponding to other particles overlapped or in close proximity. Furthermore, it would compromise the accuracy of the CLD obtained and the subsequent size estimation from the CLD inversion. For particle content estimation, the crowded particle population results in different impacts on the PSDs and CLDs. Figures 4(a) and (b) compare the total number of objects detected, and the number of objects in focus, over 10,000 frames for the in-line imaging method. Note an object is defined as an image of a substance with well-defined edges and can correspond to either a fully or partially in-focus particle, or a cluster of overlapping particles.[24] The number of objects in Figures 4(a) and (b) can also be expressed as a function of particle number density, as shown in Figures 4 (c) and (d). The particle number density is defined as the number of particles in a unit mass of suspension; the number of particles is calculated by dividing the total volume of particles to the volume of one particle. Given the presence of ellipsoids in most of the particle size groups, the calculation of particle volume assumes this shape and the volume of ellipsoids for each size group is calculated using the median particle volume obtained by the off-line imaging method. 
Figure 4(c) shows that the number of objects detected responds linearly to an increase of number density; however, the number of objects in focus in Figure 4(d) follows a downward trend for particles number density larger than 10,000 particles $\mathrm{g}^{-1}$. The observation is independent of the particle size group and can be attributed to overlapping particles at higher concentrations. Overlapped particles are more likely to be discarded by partially falling outside the image frame and together with the deteriorated contrast of the images for high concentrations, reduces the representativeness of the results from in-line imaging. Therefore, the particle number density of 10,000 particles $\mathrm{g}^{-1}$ can be considered as the upper limit for quantitative in-line image analysis on solid loading. Previous studies by Agimelen et al. [7] and Cardona et al. [24] identified challenges in estimating PSD from data captured with PVM due to camera resolution limits and out of focus rejection requirements. However, limits of particle number density which can be used for an accurate analysis was not identified. This parameter allows defining the required image contrast and resolution for the future design of in-line imaging equipment and extends the applicability of the image analysis algorithm. It also informs the user of the experimental conditions where other PAT techniques need to be sought for more reliable results.

Similarly, an increase in total CL counts from unweighted CLDs is observed when increasing solid loading and particle number density, as shown in Figure 5. For smaller particles, Figure 5(a) shows reduced linearity between the total CL counts and solid loading as a result of the overcrowded suspensions and concomitant reduction in signal resolution in CL measurements. In suspensions with high solid content, the incident light for CL measurements can undergo several light scattering events that produce a diffused reflected signal, which in turn is background noise that interferes with the determination of CLs by reducing the number of counts. Compared to Figure 4(d), Figure 5(b) shows improved linearity between particle number density and total CL count. Also, Figure 5 does not indicate an upper detection limit 
for CL counts but reveals the potential for utilising CL counts as an indicator of solid content in a suspension.

\subsection{Estimating particle size and concentration from CLD using PLSR analysis}

As discussed, the PSD obtained is strongly dependent on the measurement technique, and there is no intuitive way to accurately describe the "true" particle size distribution or concentration of a system, particularly for in-line methods. Although the quality of the PSDs obtained through in-line imaging can be improved by increasing the number of frames captured or by optimising the optical design of the probe, the results are an adequate representation within the range of applicability $\left(<10,000\right.$ particles $\left.\mathrm{g}^{-1}\right)$ without further need for data processing. However, important limitations have been identified for PSDs obtained through inversion of CLDs due to chord splitting, which limits the scope of use of this method for particle size estimation. As discussed previously, the chord splitting is often observed when analysing large and smooth transparent particles. Nevertheless, it can still affect the CLDs from crystals of different shapes or surface roughness, despite the reduction of specular reflection.[32] Despite the performance limitations of the inversion method, a good correlation still exists between the changes in CL counts and positions in CLDs, and particle size and concentration. Therefore, it is highly desirable to explore alternatives to physical-based analysis approaches for estimating particle information which minimise potentially misleading results.

PLSR analysis is a multivariate analysis (MVA) method which utilises an inferential statistical approach for analysing changes in variables and identifying hidden features in complex data. PLSR is widely adopted in spectroscopic studies where the changes of chemical or physical species can be related to the changes in spectral features, including intensity and peak position.[44-46] Since the shape of CLDs resembles the response observed in spectral analysis, PLRS is investigated as an alternative to the inversion method. 
The unweighted and square-weighted CLDs used for PLSR analysis are shown in Figure 6. Considering the CLDs from PS630-800 correspond mostly to the split chord, all CLDs from this size group are excluded from the analysis. As the CL count varies significantly from 20 up to 700 , it could be challenging for the PLSR model to capture features in CLDs related to particle size. The pre-processing approaches, SNV and logarithmic transformation (log), were selected to convert the CLDs into a comparable y-scale and improve the linear relationship between the CLDs and the parameters of interest.

Table 3 summarises PLSR models for estimating particle size using CLDs pre-processed differently. The number of latent variables (LVs) for each model was chosen based on the minimum number required to achieve the merit of the model. SNV pre-processing contributes to a noticeable improvement in the models through either reducing the RMSECV or the number of LVs required. The combination of both pre-processing methods, however, results in the poorest performance. Although the log transformation brings the CL counts into a comparable scale, it also makes the CLD shape and position less distinguishable between different particle size groups, limiting the improvement to the model performance. In this case, only a small improvement is obtained, and the model requires using more LVs. Since the SNV is a normalisation method to regularise the variation in the data, the pre-processed CLDs become more consistent within the same particle size group resulting in improved model performance.

Models using SNV pre-processed CLDs consistently return the best model performance for both types of CLDs, although the improvement is limited to only slightly reduced RMSECV and $\mathrm{R}^{2}$, as well as fewer LVs required. To further differentiate model performance, the RMSECV curves for models established through SNV pre-processed unweighted and weighted CLDs are compared, as shown in Figure 7(a). Both models exhibit similar behaviour with a clear indication of the number of LVs for calibration. Considering square-weighted CLDs require one less $\mathrm{LV}$, the model is chosen as the best model for the particle size estimation, 
giving an RMSECV of $27.6 \mu \mathrm{m}$ and an $\mathrm{R}^{2}$ of 0.950 . Note Figure $7(\mathrm{~d})$ and (e) are shown in the linear chord length scale to present the features of CLDs and corresponding LVs. The format in which the CLs are expressed does not affect model performance.

Similar analysis steps were applied to establish PLSR models for estimating solid loadings in the suspension. As the effect of solid loading mainly manifests in CLDs as an increase in counts for all CL groups, the large difference in CL counts can be utilised by the PLSR model. However, as Figure 3(c) indicated, the increase in solid loading also alters the shape of CLDs, mainly by shifting and broadening the main CL peak, which in turn reduces the resolution of the minor CL peak or shoulder. Since such changes in CLDs are dependent on the particle size group, it could add complexity to the PLSR analysis.

Table 4 summarises PLSR models established for estimating particle concentration using different pre-processed CLDs; similar performance was obtained for all models established on pre-processed CLDs. The pre-processing methods suppress the magnitude difference in the CLDs, which subsequently enhances the influence of non-systematic changes in the CLD shape. The best PLSR model results in an RMSECV of $1.06 \mathrm{wt} \%$ and $\mathrm{R}^{2}$ of $0.897 \mathrm{using}$ unweighted CLDs without pre-processing. Similar RMSECV and $\mathrm{R}^{2}$ are obtained from squareweighted CLDs, but the model requires more LVs.

Results from the best PLSR model on estimating solid loading are shown in Figure 8. The RMSECV curve in Figure 8(a) shows a clear decrease in the error after using more than 2 LVs and reaches a plateau when 5 LVs or more are used. As observed in Figure 3(c) and (d), the increase of solid loading not only increases CL counts but also causes a shift towards smaller lengths among samples in the same particle size group. Figure 8(d) shows all CLDs used for the PLSR analysis. The PLSR model results in loading curves exhibiting oscillating features, as those for LV2-5 in Figure 8(e1) and (e2). These features, when combined with the loading 
curve of LV1, can resemble the shape of CLDs for different particle size groups, and describe the change in the CLD due to the increase of solid content. The features in Figure 8(e1) and (e2) are typically observed when developing multivariate regression models to describe a system where the measured profile shifts with the modelled condition, such as models describing the temperature-dependent shift of the water absorption peak.[47] The changes in CL counts in each particle group are captured by the PLSR model, showing good linearity in the prediction with residuals scattered randomly, as shown in Figures $8(\mathrm{~b})$ and (c). The issue of CLD affected by the overcrowded particle population or chord splitting does not lead to any apparent outliers in the prediction and residual plots, although samples of the extreme conditions may be found to have slightly larger residuals.

Overall, the results from the PLSR analysis are encouraging. Together with the complex response of CL to the parameters of interest and the different mixing ratios of ellipsoidal to spherical particles, the complicated information contained in the small dataset under consideration is effective in demonstrating the potential for using MVA-based quantitative analysis for in-line particle measurements. Other in-line particle analysers can also benefit from a similar approach if the sample is within specific performance limits; in the case of in-line imaging, the particle number density limit is 10,000 particles $\mathrm{g}^{-1}$. The results of the comparative analysis indicate a viable methodology for the full extraction of the information captured inline. Unexpected changes in CLD such as chord splitting, [40, 43, 48, 49] non-uniform backscattering $[32,43]$ and increasing particle number density, which cannot be modelled accurately by physical-based inversion method, can be interpreted systematically by multivariate analysis.

\section{Conclusions}

The results of a comparative study of the method of analysis and the performance of in-line and off-line imaging, in-line chord length measurement and laser diffraction techniques to 
quantify particle size and concentration in suspensions are presented. The presence of nonspherical particles in the mixture can be captured by PSDs from off-line measurements and inline imaging methods. The mixture of spherical and ellipsoidal particles in the suspension increases the complexity and the challenge in deriving the information contained in CLDs, translating in a reduction in the sensitivity of the CLD inversion method at estimating particle size. The in-line methods also show limited performance in estimating larger particles $(>300$ $\mu \mathrm{m})$ due to split chords in CLD measurements and particles being more likely to fall on the image frame for in-line imaging. On the other hand, quantitating particle concentration can be achieved using particle and CL counts from in-line methods. The performance is only reliable for suspensions of a particle density under 10,000 particles $\mathrm{g}^{-1}$; above such a limit, a non-linear response is observed in both in-line imaging and CL measurements due to overlapping particles.

The study also evaluates the use of PLSR analysis as an alternative approach to quantitate particle size and concentration. Similar PLSR model performance is obtained for models established on the weighted and unweighted CLDs. The best models result in an RMSECV of $27 \mu \mathrm{m}$ and $1.06 \mathrm{wt} \%$ for estimating particle size and concentration, respectively.

PLSR models better describe the changes in CLDs with increasing solid loading; improved linearity is observed when compared to the estimation using the CL counts. While the evaluation established PLSR models for median particle size, the findings encourage further development of MVA-based analysis for estimating PSD. The results indicate a potentially promising approach to obtaining quantitative particle information in-line without the need to apply inversion algorithms for CLD analysis. 


\section{Acknowledgements}

The authors would like to thank EPSRC and the Doctoral Training Centre in Continuous Manufacturing and Crystallisation (Grant Ref: EP/K503289/1 and Intelligent Decision Support and Control Technologies for Continuous Manufacturing of Pharmaceuticals and Fine Chemicals (Grant Ref: EP/K014250/1) and Centre for Innovative Manufacturing in Continuous Manufacturing and Crystallisation (Grant Ref: EP/I033459/1) for funding this work.

The authors would like to acknowledge that this work was carried out in the CMAC National Facility supported by UKRPIF (UK Research Partnership Fund) award from the Higher Education Funding Council for England (HEFCE) (Grant Ref: HH13054).

All data underpinning this publication are openly available from the University of Strathclyde at https://doi.org/10.15129/2f3bdb7a-a19c-4e6c-86d8-368da31a2355

\section{References}

[1] L.X. Liu, I. Marziano, A.C. Bentham, J.D. Litster, E.T.White, T. Howes, Effect of particle properties on the flowability of ibuprofen powders, Int. J. Pharm., 362 (2008) 109-117.

[2] B. Ahmed, C.J. Brown, T. McGlone, D.L. Bowering, J. Sefcik, A.J. Florence, Engineering of acetaminophen particle attributes using a wet milling crystallisation platform, Int. J. Pharm., 554 (2019) 201-211.

[3] H.G. Morrison, W. Tao, W. Trieu, S.D. Walker, S. Cui, S. Huggins, K. Nagapudi, Correlation of Drug Substance Particle Size Distribution with Other Bulk Properties to Predict Critical Quality Attributes, Org. Process Res. Dev., 19 (2015) 1076-1081.

[4] P. Narayan, B.C. Hancock, The relationship between the particle properties, mechanical behavior, and surface roughness of some pharmaceutical excipient compacts, Mater. Sci. Eng. A, 355 (2003) 24-36.

[5] A.F.T. Silva, A. Burggraeve, Q. Denon, P. Van der Meeren, N. Sandler, T. Van Den Kerkhof, M. Hellings, C. Vervaet, J.P. Remon, J.A. Lopes, T. De Beer, Particle sizing measurements in pharmaceutical applications: Comparison of in-process methods versus offline methods, Eur. J. Pharm. Biopharm., 85 (2013) 1006-1018. 
[6] A. Abbas, D. Nobbs, J.A. Romagnoli, Investigation of on-line optical particle characterization in reaction and cooling crystallization systems. Current state of the art, Meas. Sci. Technol., 13 (2002) 349-356.

[7] O.S. Agimelen, V. Svoboda, B. Ahmed, J. Cardona, J. Dziewierz, C.J. Brown, T. McGlone, A. Cleary, C. Tachtatzis, C. Michie, A.J. Florence, I. Andonovic, A.J. Mulholland, J. Sefcik, Multi-sensor inline measurements of crystal size and shape distributions during high shear wet milling of crystal slurries, Adv. Powder Technol., 29 (2018) 2987-2995.

[8] A.J. O'Neil, R.D. Jee, A.C. Moffat, Measurement of the percentage volume particle size distribution of powdered microcrystalline cellulose using reflectance near-infrared spectroscopy, Analyst, 128 (2003) 1326-1330.

[9] A. J. O’Neil, R. D. Jee, A. C. Moffat, The application of multiple linear regression to the measurement of the median particle size of drugs and pharmaceutical excipients by nearinfrared spectroscopy, Analyst, 123 (1998) 2297-2302.

[10] L.J. Bellamy, A. Nordon, D. Littlejohn, Real-time monitoring of powder mixing in a convective blender using non-invasive reflectance NIR spectrometry, Analyst, 133 (2008) 5864.

[11] ISO 13320:2009 Particle Size Analysis-Laser Diffraction Methods. Part 1: General Principles, 2009.

[12] H.G. Merkus, Particle Size Measurements: Fundamentals, Practice, Quality, 1 ed., Springer Netherlands, Springer, 2009.

[13] D. Wiegel, G. Eckardt, F. Priese, B. Wolf, In-line particle size measurement and agglomeration detection of pellet fluidized bed coating by Spatial Filter Velocimetry, Powder Technol., 301 (2016) 261-267.

[14] A. Burggraeve, T. Van Den Kerkhof, M. Hellings, J.P. Remon, C. Vervaet, T. De Beer, Evaluation of in-line spatial filter velocimetry as PAT monitoring tool for particle growth during fluid bed granulation, Eur. J. Pharm. Biopharm., 76 (2010) 138-146.

[15] V. Kumar, M.K. Taylor, A. Mehrotra, W.C. Stagner, Real-Time Particle Size Analysis Using Focused Beam Reflectance Measurement as a Process Analytical Technology Tool for a Continuous Granulation-Drying-Milling Process, AAPS Pharm. Sci. Tech., 14 (2013) 523530.

[16] O.S. Agimelen, P. Hamilton, I. Haley, A. Nordon, M. Vasile, J. Sefcik, A.J. Mulholland, Estimation of particle size distribution and aspect ratio of non-spherical particles from chord length distribution, Chem. Eng. Sci., 123 (2015) 629-640. 
[17] M. Li, D. Wilkinson, Determination of non-spherical particle size distribution from chord length measurements. Part 1: Theoretical analysis, Chem. Eng. Sci., 60 (2005) 3251-3265.

[18] J. Worlitschek, T. Hocker, M. Mazzotti, Restoration of PSD from Chord Length Distribution Data using the Method of Projections onto Convex Sets, Part. Part. Syst. Charact., 22 (2005) 81-98.

[19] W. Yu, K. Erickson, Chord length characterization using focused beam reflectance measurement probe - methodologies and pitfalls, Powder Technol., 185 (2008) 24-30.

[20] N. Sandler, Photometric imaging in particle size measurement and surface visualization, Int. J. Pharm., 417 (2011) 227-234.

[21] A. Kumar, J. Dhondt, F. De Leersnyder, J. Vercruysse, V. Vanhoorne, C. Vervaet, J.P. Remon, K.V. Gernaey, T. De Beer, I. Nopens, Evaluation of an in-line particle imaging tool for monitoring twin-screw granulation performance, Powder Technol., 285 (2015) 80-87.

[22] A.K. Rajagopalan, J. Schneeberger, F. Salvatori, S. Bötschi, D.R. Ochsenbein, M.R. Oswald, M. Pollefeys, M. Mazzotti, A comprehensive shape analysis pipeline for stereoscopic measurements of particulate populations in suspension, Powder Technol., 321 (2017) 479-493. [23] Z.K. Nagy, G. Fevotte, H. Kramer, L.L. Simon, Recent advances in the monitoring, modelling and control of crystallization systems, Chem. Eng. Res. Des., 91 (2013) 1903-1922. [24] J. Cardona, C. Ferreira, J. McGinty, A. Hamilton, O.S. Agimelen, A. Cleary, R. Atkinson, C. Michie, S. Marshall, Y.-C. Chen, J. Sefcik, I. Andonovic, C. Tachtatzis, Image analysis framework with focus evaluation for in situ characterisation of particle size and shape attributes, Chem. Eng. Sci., 191 (2018) 208-231.

[25] J. Huang, G. Kaul, J. Utz, P. Hernandez, V. Wong, D. Bradley, A. Nagi, D. O’Grady, A PAT Approach to Improve Process Understanding of High Shear Wet Granulation Through InLine Particle Measurement Using FBRM C35, J. Pharm. Sci., 99 (2010) 3205-3212.

[26] R. Beck, K. Svinning, A. Häkkinen, D. Malthe-Sørenssen, J.-P. Andreassen, Analysis of Filtration Characteristics for Compressible Polycrystalline Particles by Partial Least Squares Regression, Sep. Sci. Technol., 45 (2010) 1196-1208.

[27] T. Togkalidou, R.D. Braatz, B.K. Johnson, O. Davidson, A. Andrews, Experimental design and inferential modeling in pharmaceutical crystallization, AIChE J., 47 (2001) 160168.

[28] G.J. Emidio, Spatially and angularly resolved diffuse reflectance spectroscopy for in-situ monitoring of suspension polymerisation reactions, Ph.D. thesis, Department of Chemical and Process Engineering, University of Strathclyde, 2017. 
[29] J. Cardona, C. Tachtatzis, ImagingApp: image analysis framework for particle size and shape characterisation, 2018. https://doi.org/10.15129/99c4558f-f59e-4be8-80e2$\underline{b 0425178332 b}$

[30] O.S. Agimelen, A. Jawor-Baczynska, J. McGinty, J. Dziewierz, C. Tachtatzis, A. Cleary, I. Haley, C. Michie, I. Andonovic, J. Sefcik, A.J. Mulholland, Integration of in situ imaging and chord length distribution measurements for estimation of particle size and shape, Chem. Eng. Sci., 144 (2016) 87-100.

[31] O.S. Agimelen, A.J. Mulholland, J. Sefcik, Software for transforming measured chord length distribution data to particle size distribution, 2018. https://doi.org/10.15129/c147b4c4$\underline{6 e 2 e-43 b d-b d f d-f d 889 e f 2 d 5 e e}$

[32] J. Heinrich, J. Ulrich, Application of Laser-Backscattering Instruments for In Situ Monitoring of Crystallization Processes - A Review, Chem. Eng. Technol., 35 (2012) 967-979. [33] K. Sommer, 40 Years of Presentation Particle Size Distributions-Yet Still Incorrect?, Part. Part. Syst. Charact., 18 (2001) 22-25.

[34] Y.-C. Chen, D. Foo, N. Dehanov, S.N. Thennadil, Spatially and angularly resolved spectroscopy for in-situ estimation of concentration and particle size in colloidal suspensions, Anal. Bioanal. Chem., 409 (2017) 6975-6988.

[35] R.J. Barnes, M.S. Dhanoa, S.J. Lister, Standard Normal Variate Transformation and DeTrending of Near-Infrared Diffuse Reflectance Spectra, Appl. Spectrosc., 43 (1989) 772-777. [36] N.R.J. Fieller, E.C. Flenley, W. Olbricht, Statistics of Particle Size Data, J. Royal Stat. Soc. C, 41 (1992) 127-146.

[37] P. Bowen, Particle Size Distribution Measurement from Millimeters to Nanometers and from Rods to Platelets, J. Disper Sci. Technol., 23 (2002) 631-662.

[38] R. Hogg, A Spheroid Model for the Role of Shape in Particle Size Analysis, KONA Powder Part. J., 32 (2015) 227-235.

[39] P. Hamilton, D. Littlejohn, A. Nordon, J. Sefcik, P. Slavin, Validity of particle size analysis techniques for measurement of the attrition that occurs during vacuum agitated powder drying of needle-shaped particles, Analyst, 137 (2012) 118-125.

[40] A. Ruf, J. Worlitschek, M. Mazzotti, Modeling and Experimental Analysis of PSD Measurements through FBRM, Part. Part. Syst. Charact., 17 (2000) 167-179.

[41] K. Vay, W. Frieß, S. Scheler, Understanding reflection behavior as a key for interpreting complex signals in FBRM monitoring of microparticle preparation processes, Int. J. Pharm., 437 (2012) 1-10. 
[42] R.G. Sparks, C.L. Dobbs, The Use of Laser Backscatter Instrumentation for the on-line measurement of the particle size distribution of emulsions, Part. Part. Syst. Charact., 10 (1993) 279-289.

[43] N. Kail, H. Briesen, W. Marquardt, Advanced Geometrical Modeling of Focused Beam Reflectance Measurements (FBRM), Part. Part. Syst. Charact., 24 (2007) 184-192.

[44] W.B. Lee, E. Widjaja, P.W.S. Heng, L.W. Chan, Near infrared spectroscopy for rapid and in-line detection of particle size distribution variability in lactose during mixing, Int. J. Pharm., 566 (2019) 454-462.

[45] T. De Beer, A. Burggraeve, M. Fonteyne, L. Saerens, J.P. Remon, C. Vervaet, Near infrared and Raman spectroscopy for the in-process monitoring of pharmaceutical production processes, Int. J. Pharm., 417 (2011) 32-47.

[46] B. Nagy, A. Farkas, E. Borbás, P. Vass, Z.K. Nagy, G. Marosi, Raman Spectroscopy for Process Analytical Technologies of Pharmaceutical Secondary Manufacturing, AAPS Pharm. Sci. Tech., 20 (2018) 1.

[47] B. Czarnik-Matusewicz, S. Pilorz, Study of the temperature-dependent near-infrared spectra of water by two-dimensional correlation spectroscopy and principal components analysis, Vib. Spectrosc, 40 (2006) 235-245.

[48] M.R. Abu Bakar, Z.K. Nagy, C.D. Rielly, Investigation of the Effect of Temperature Cycling on Surface Features of Sulfathiazole Crystals during Seeded Batch Cooling Crystallization, Cryst. Growth Des, 10 (2010) 3892-3900.

[49] F. Czapla, N. Kail, A. Öncül, H. Lorenz, H. Briesen, A. Seidel-Morgenstern, Application of a recent FBRM-probe model to quantify preferential crystallization of dl-threonine, Chem. Eng. Res. Des., 88 (2010) 1494-1504. 


\section{Figure 1}
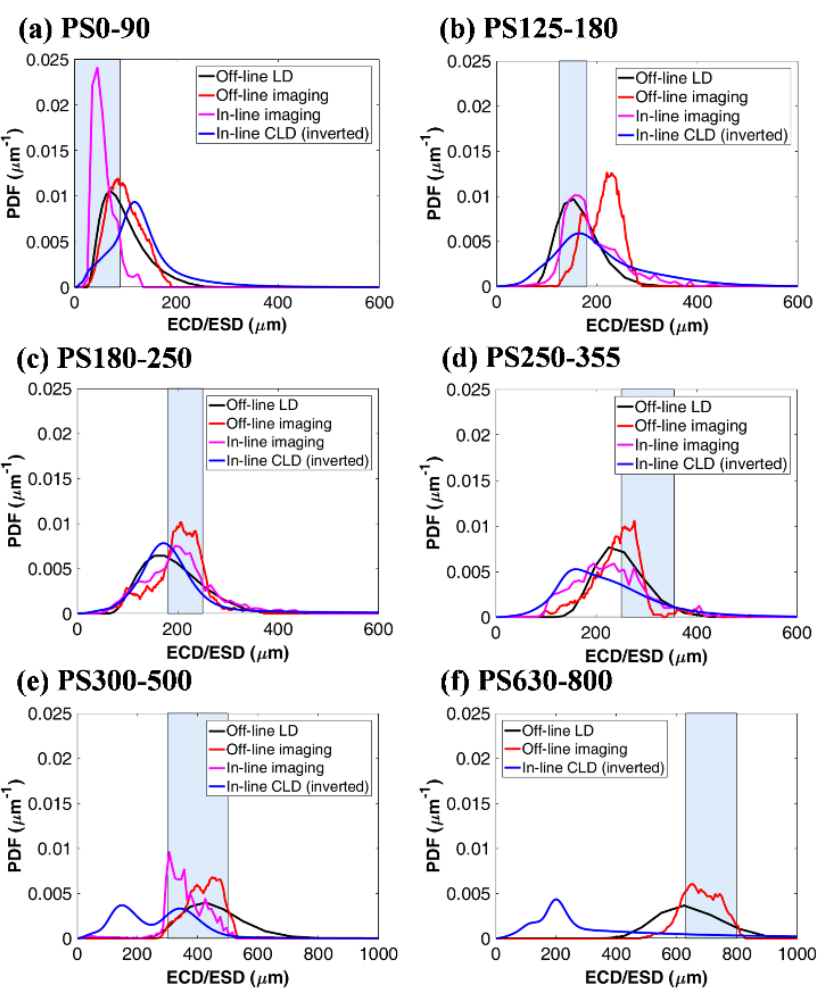

Figure 1: PDF from different techniques used in this study: (a)-(f) correspond to the groups of the smallest particles to the largest particles in Table 1. The shaded size ranges correspond to those defined by sieving. Insufficient data was captured from the in-line imaging method for PS630-800. 


\section{Figure 2}

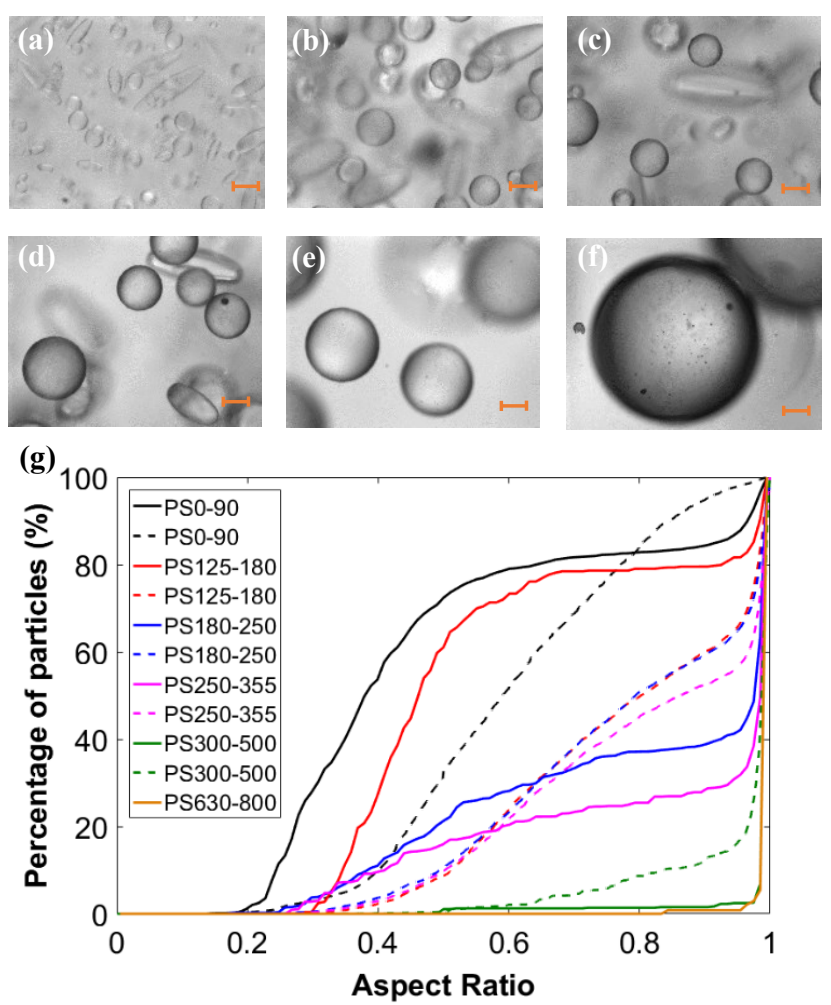

Figure 2: (a)-(f) Typical in-line images of polystyrene beads at $10 \mathrm{wt} \%$ of (a) PS0-90, (b) PS125-180, (c) PS180-250, (d) PS250-355, (e) PS300-500 and (f) PS630-800. The scale bar indicates $100 \mu \mathrm{m} .(\mathrm{g})$ Cumulative distributions of aspect ratio obtained from off-line imaging (solid lines) and in-line imaging (dashed lines) analysis. 


\section{Figure 3}
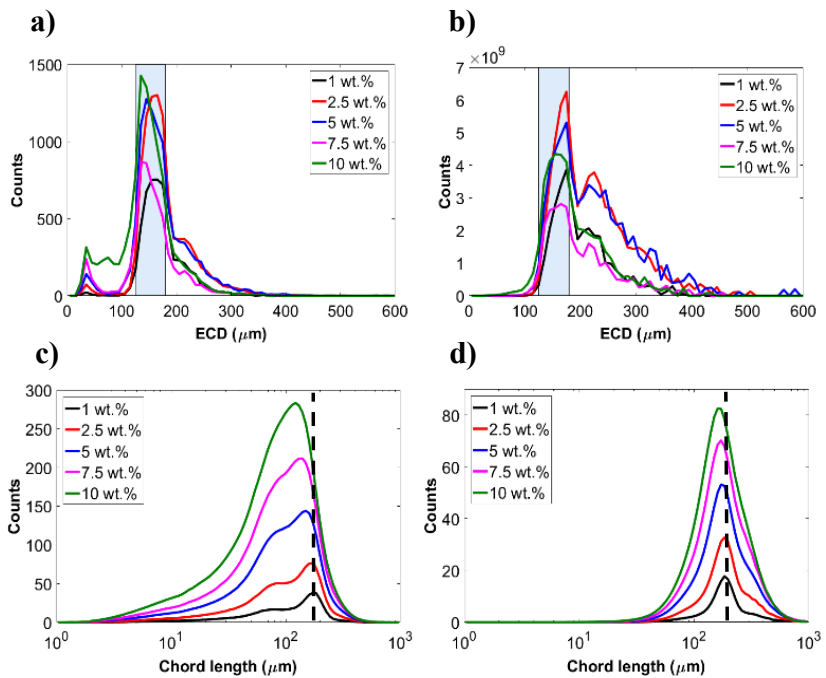

Figure 3: Effect of solid loading of PS125-180 expressed in PSDs from in-line image analysis, and CLD. a) and b) are particle counts of the number- and volume-weighted ECD obtained from imaging, and the shaded area corresponds to the size range defined by sieving. c) and d) are unweighted and square-weighted CLDs, respectively, and the dashed line corresponds to the chord length of maximum count at $1 \mathrm{wt} . \%$. 


\section{Figure 4}

a)

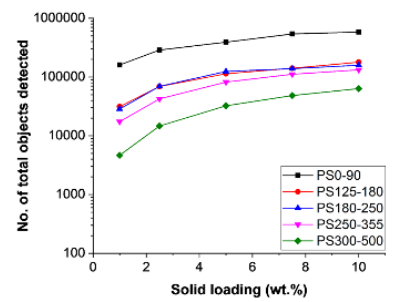

c)

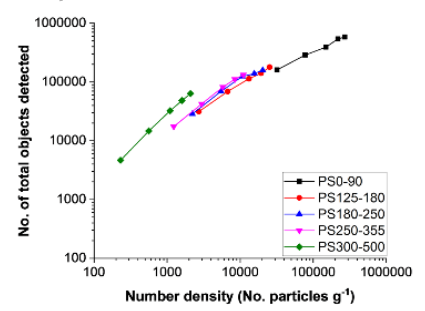

b)

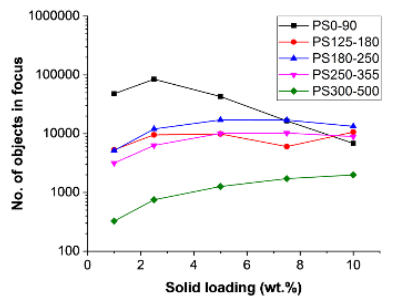

d)

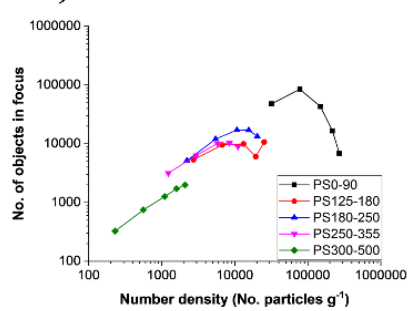

Figure 4: a) Total number of objects detected and b) number of objects in focus by in-line imaging at different particle sizes and solid loadings. c) and d) express the total number of objects in a) and b), respectively, in terms of particle number density. 


\section{Figure 5}

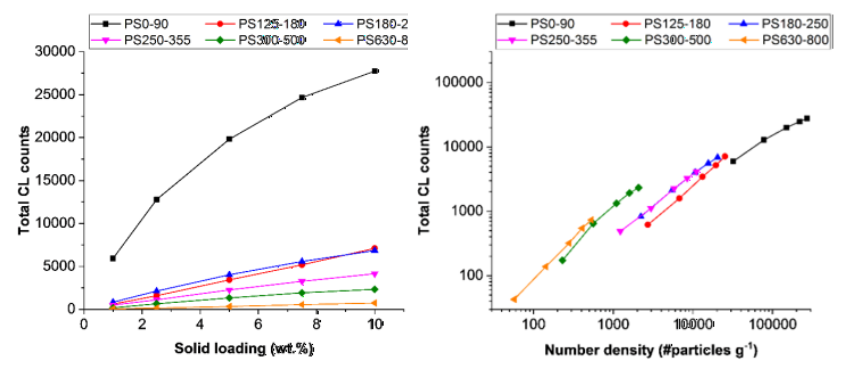

Figure 5: Changes in total number of CL counts with a) solid loading and b) number of particles per g of sample, for all samples. 


\section{Figure 6}
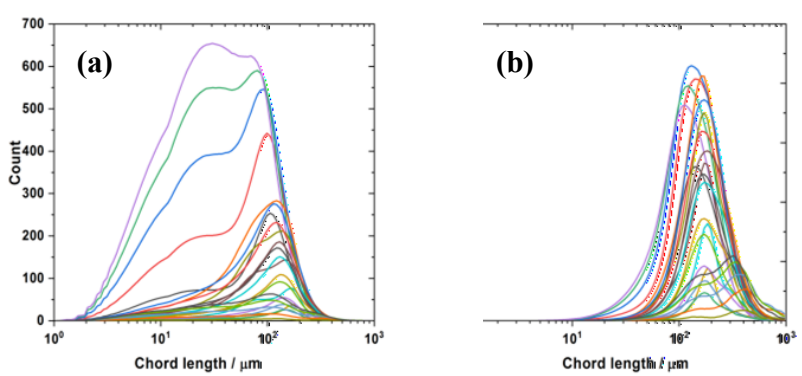

Figure 6: (a) unweighted and (b) square-weighted CLDs used for multivariate regression analysis. 


\section{Figure 7}
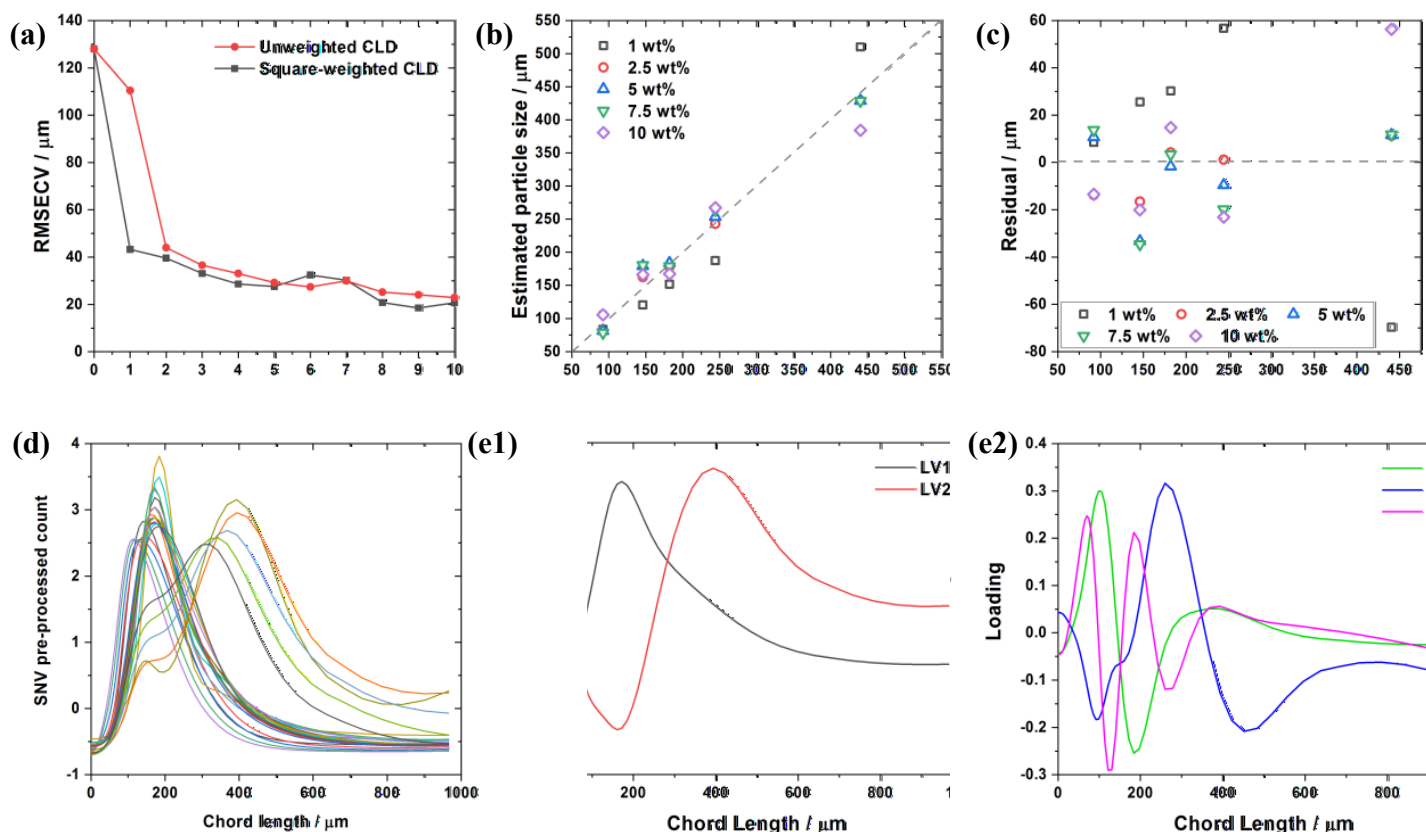

(e1)
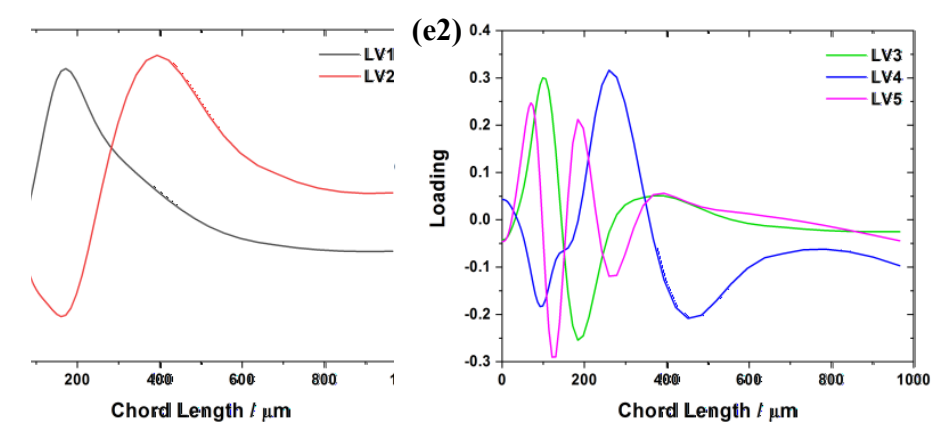

Figure 7: (a) RMSECV curve of models built on unweighted and square-weighted CLDs preprocessed by SNV, (b)-(e) correspond to the model built on the SNV pre-processed, squaredweighted CLDs. (b) and (c) are the prediction plot and residual plot. (d) presents the preprocessed CLDs, and (e1) and (e2) correspond to the LV curves of the model. 


\section{Figure 8}

(a)

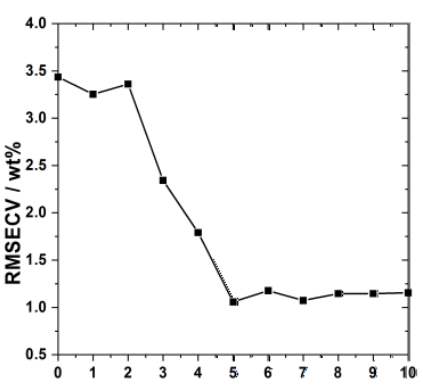

(d)

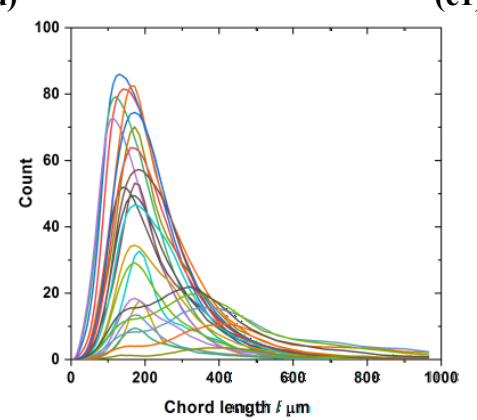

(b)

(e1)
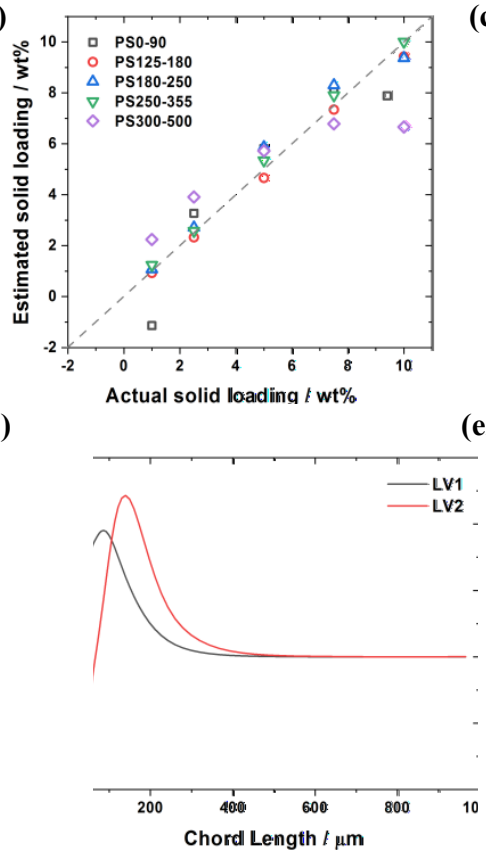

(c)

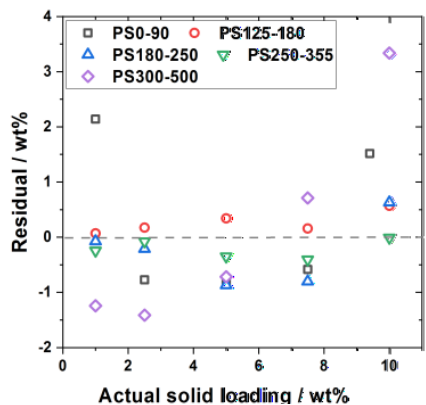

(e2)

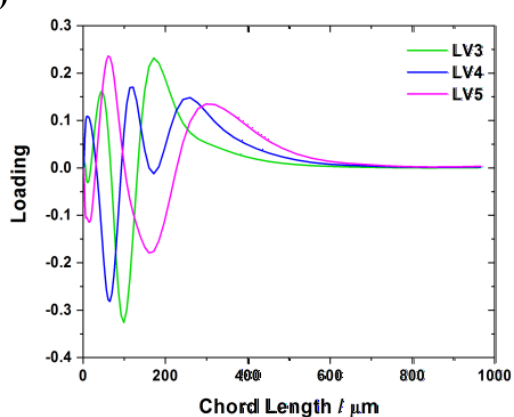

Figure 8: (a) RMSECV curve, (b) prediction plot, (c) residual plot from the PLSR model built on the unweighted CLDs (in (d)) for estimating solid loading. (e1) and (e2) to LV1-2 and LV $3-5$, respectively. 


\section{Quantification of Particle Size and Concentration Using In-line Techniques and Multivariate Analysis}

Carla Ferreira $^{1,2}$, Javier Cardona ${ }^{3}$, Okpeafoh S. Agimelen ${ }^{1,2}$, Christos Tachtatzis ${ }^{3}$, Ivan Andonovic $^{3}$, Jan Sefcik ${ }^{1,2}$ and Yi-Chieh Chen ${ }^{2 *}$

${ }^{1}$ EPSRC Future Manufacturing Research Hub for Continuous Manufacturing and Advanced

Crystallisation (CMAC), University of Strathclyde, Glasgow, United Kingdom

${ }^{2}$ Department of Chemical and Process Engineering, University of Strathclyde, Glasgow, United Kingdom

${ }^{3}$ Department of Electronic and Electrical Engineering, University of Strathclyde, Glasgow, United Kingdom

e-mail: yichieh.chen@strath.ac.uk

Tel: +441215745304

\section{S1. Experimental Setup}

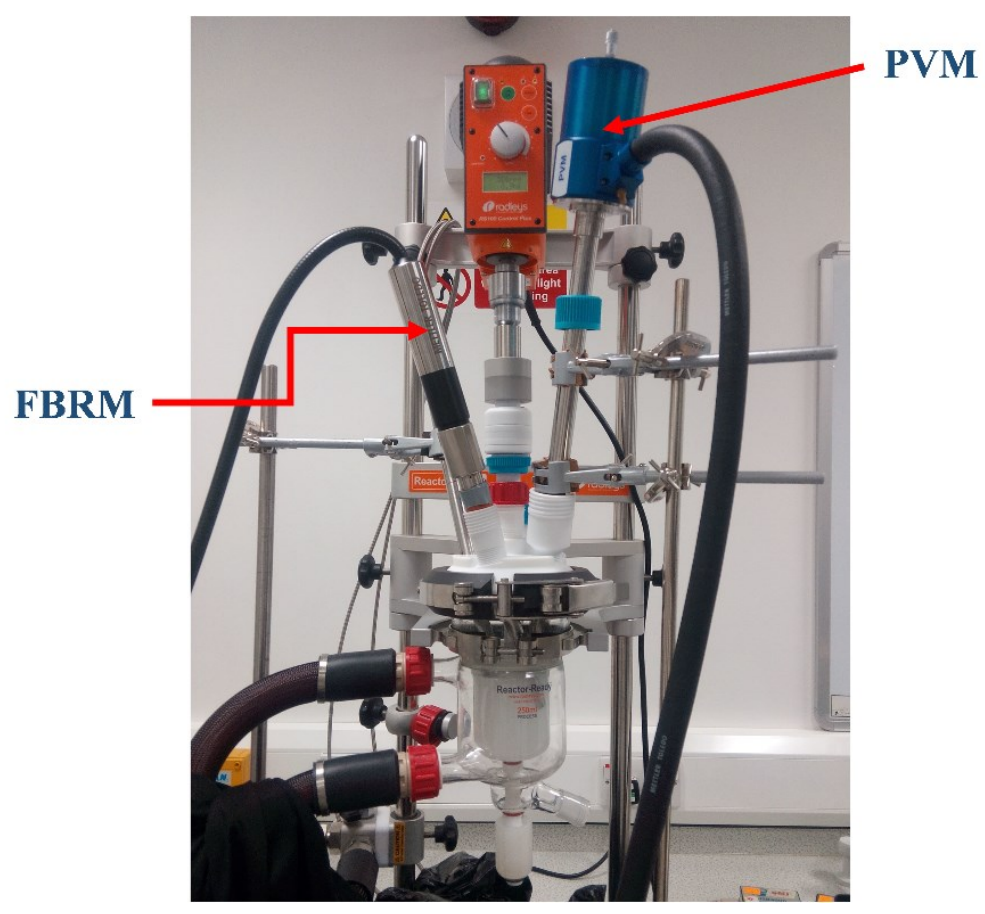

SFigure 1: Experimental setup for the in-line particle measurements. 


\section{S2. Effect of specular reflection}

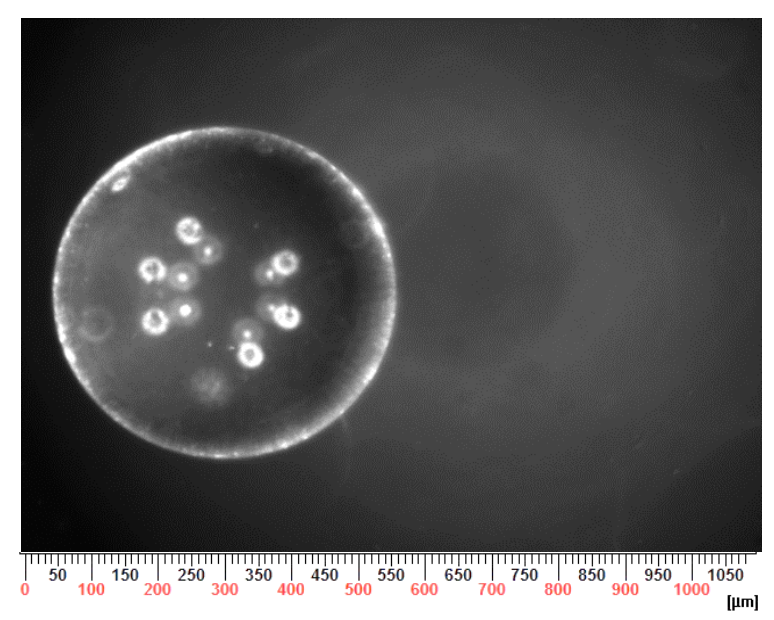

SFigure 2: A PVM image of a large, transparent particle with a smooth surface.

SFigure 2 shows a typical example of a reflected image of particle from PVM measurement. Two sets of bright dots, each consisting of 6 'dots' are clearly observed inside the particle. The larger dots in the outer set correspond to the specular reflection of the PVM's six illumination laser beams from the upper surface of the particles. The smaller dots in the inner set can be attributed to the reflected illumination from the back of the particle due to internal reflection. The image highlights the potential challenges inherent in current CL measurement. Transparent particles have been reported to yield poor size analysis in CL measurement ${ }^{1-4}$. These particles produce considerable specular reflection, or a high degree of internal reflection, of the laser incident beam for the measurement, resulting in the split of the chords. 


\section{S3. Effect of solid loading on PSD using in-line imaging method}

\section{a)}

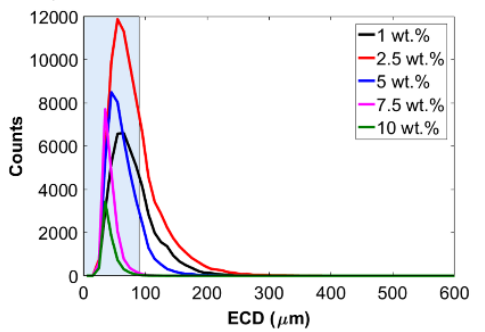

c)

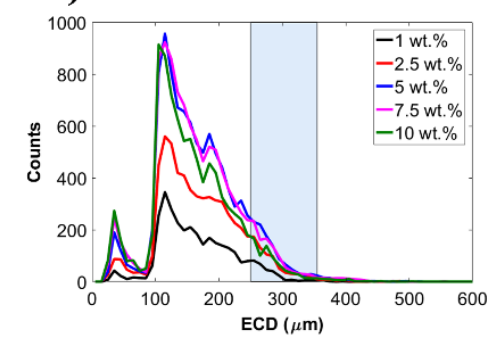

b)

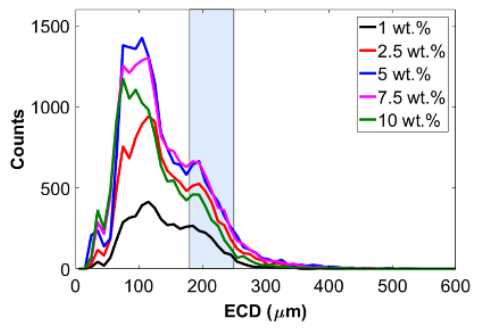

d)

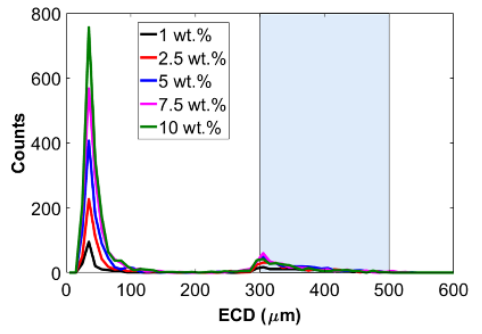

SFigure 3: Effect of solid loading on the number-weighted ECD obtained from in-line image analysis. (a)-(e) corresponds to PS0-90, PS180-250, PS250-355 and PS300-500, respectively. The shaded size range corresponds to those defined by sieving.

SFigure 3 exhibits the effect of solid loading on the number-weighted ECD for all particle size groups (except for PS630-800, where no sufficient particles are collected). Since the numberweighted distribution is sensitive to the particle count in the sample, increasing the solid loading initially increases the number of objects detected by PVM before the maximum particle number density is reached. A further increase in solid loading can overcrowd images, and particles are subsequently discarded by the image analysis algorithm. 
a1)

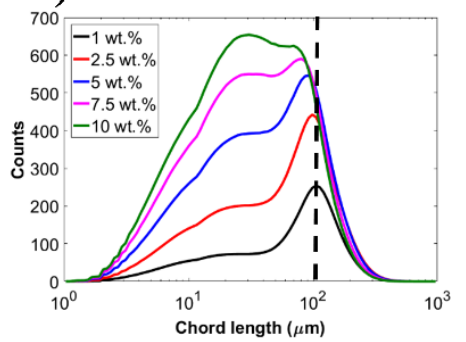

a2)

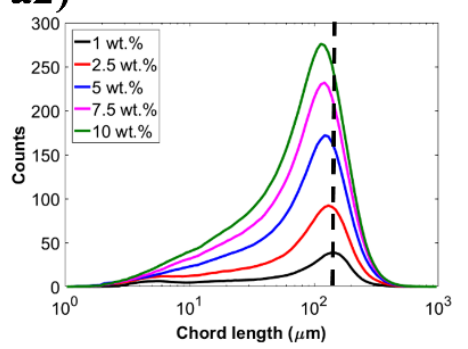

a3)

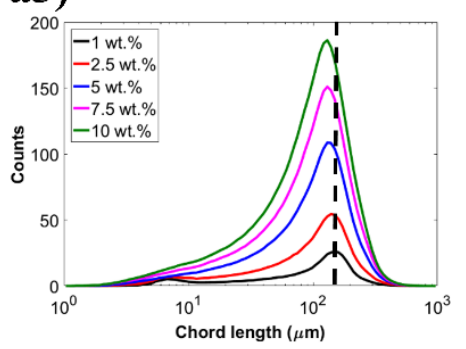

a4)

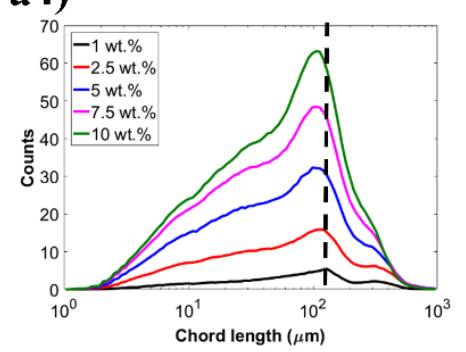

a5)

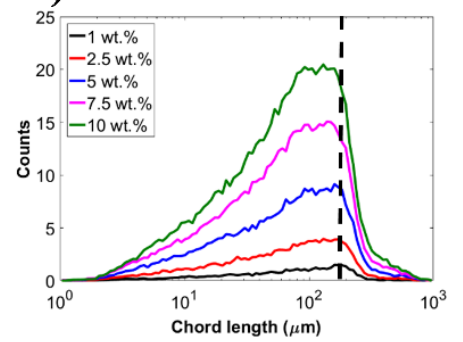

b1)

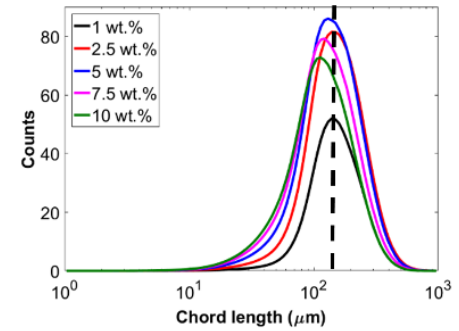

b2)

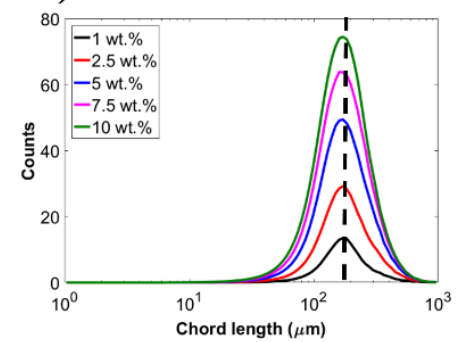

b3)

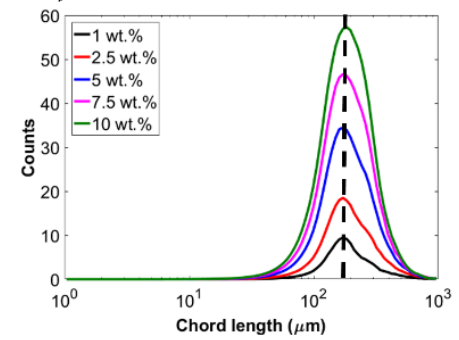

b4)

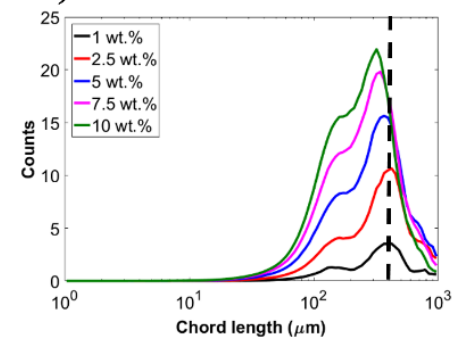

b5)

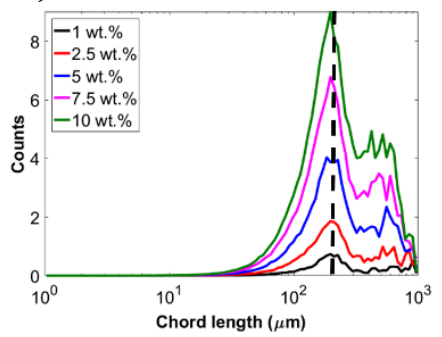

SFigure 4: Effect of solid loading on the a) unweighted and b) square-weighted CLD using (1) PS090, (2) PS180-250, (3) PS250-355, (4) PS300-500 and (5) PS630-800. The dashed line corresponds to the most frequent $\mathrm{CL}$ at $5 \mathrm{wt} \%$. 
SFigure 4 shows the effect of solid loading on the unweighted and square-weighted CLD for the different particle size ranges evaluated. In all size groups, an increase in the solid loading leads to an increase in the number of counts in unweighted CLDs, and a shift in the most frequent CL to a smaller CL is observed. The unweighted CLDs for PS0-90 in SFigure 4(a) show a peak with a shoulder whose relative count to the main peak increases with the solid loading, similar to those observed for PS125-180 in Figure 3(c). The peak at $\sim 30 \mu \mathrm{m}$ in SFigure 4(a) should be related to the diameter of the spheres and the width of ellipsoidal particles. Expressing the CL measurement in the square-weighted distribution (as observed in SFigure 4(b1)-(b5)) enhances the information related to larger chord lengths, which typically has fewer CL counts and is not easy to see in the unweighted CLD due to the lower probability of being collected.

\section{References}

1. A. Ruf, J. Worlitschek and M. Mazzotti, Modeling and experimental analysis of PSD measurements through FBRM, Part. Part. Syst. Charact., 2000, 17, 167-179.

2. K. Vay, W. Frieß and S. Scheler, Understanding reflection behavior as a key for interpreting complex signals in FBRM monitoring of microparticle preparation processes, Int. J. Pharm., 2012, 437, 1-10.

3. R. G. Sparks and C. L. Dobbs, The use of laser backscatter instrumentation for the online measurement of the particle size distribution of emulsions, Part. Part. Syst. Charact., 1993, 10, 279-289.

4. N. Kail, H. Briesen and W. Marquardt, Advanced geometrical modeling of Focused Beam Reflectance Measurements (FBRM), Part. Part. Syst. Charact., 2007, 24, $184-$ 192. 